\title{
The symptomatic breast services in a university hospital: pandemic peak compared to the pre-pandemic year and future implications
}

\author{
Gaurav Jyoti Bansal $^{1}$ (D) Zara Saleem $^{1}$
}

Received: 14 August 2021 / Accepted: 24 December 2021 / Published online: 5 January 2022

(c) The Author(s), under exclusive licence to Royal Academy of Medicine in Ireland 2022

\begin{abstract}
Background COVID-19 infection led to a substantial overhaul of the symptomatic breast services within the UK.

Aim The purpose of this study was to evaluate the pattern of primary care referrals to the symptomatic one-stop clinic during the pandemic. This study also provides a snapshot of the workings of symptomatic breast services and the scope for improvements.

Methods The data points were collected for 1 month during the peak of the pandemic (April 2020) and compared to corresponding data points for the same month in the previous year (April 2019). This was compared to the monthly data from Wales Cancer Network (WCN) data source. A hundred patients from each month over 2 years were evaluated to get a snapshot into the working of the breast clinic.

Results A total of 516 patients were referred from primary care or General Practitioners (GPs), and were seen in the Hospital 'one-stop breast clinic' in April 2019. This number dropped to 330 patients during the peak of the pandemic in April 2020. Ninety percent of referrals from the GP were urgent suspected cancers or urgent referrals. This trend of referrals did not change over 2 years. There was a 5\% and 7\% cancer diagnosis rate in 2020 and 2019, respectively.

Conclusions Most patients were referred from GP as 'urgent' or 'urgent suspected cancer'. The cancer diagnosis rate reduced from 7 to 5\% during the pandemic peak but the number of 'worried well' patients did not reduce. The total number of referrals reduced, which is predictive of increased demand in the future. The authors have suggested ways to meet this demand.
\end{abstract}

Keywords Breast cancer · COVID-19 · General practitioners · One-stop breast clinics $\cdot$ Primary care $\cdot$ Radiology

\section{Introduction}

COVID-19 infection was classified as a pandemic in March 2020 by the World Health organisation(WHO) [1, 2]. This decision led to widespread changes in the provision of healthcare arrangements in the United Kingdom (UK). The provision of breast cancer services had significant implications, with many centres pausing routine breast screening services. There was a substantial overhaul of the symptomatic breast services, as a national message circulated from the government to stay at home, particularly for the frail and elderly [3]. The Association of Breast Surgeons (ABS) advised all new referrals to be scrutinised for COVID, and

Gaurav Jyoti Bansal

gjbansal@gmail.com

1 The Breast Centre, Llandough University Hospital, Cardiff and Vale University Health Board, Penarth CF64 2XX, UK virtual consultations offered to patients for low-risk breast symptoms when possible [4].

The purpose of this study was to evaluate how the pattern of primary care referrals to the symptomatic one-stop clinic changed during the pandemic peak and directly compare this to pre-pandemic practice. This study hopes to inform and evaluate the system of symptomatic breast services within the UK. This study also provides a snapshot of the workings of symptomatic breast services and the scope for improvements. This study aims to predict the future demand of primary care referrals and match demand with optimum capacity.

\section{Methods}

This retrospective study was performed and registered as part of the medical student selected component (SSC) project for 2021. The informed consent requirement was 
waived as per departmental protocol. Data was retrospectively collected and anonymised. The data points were collected for 1 month during the peak of the pandemic (April 2020) and compared to corresponding data points for the same month in the previous year (April 2019). Data was collected from the hospital Picture Archiving and Communication System (PACS) and Welsh Clinical Portal (WCP). This was compared to the monthly data from Wales Cancer Network (WCN) data source [5]. Exclusion criteria included screening patients, patients referred due to incidental imaging findings performed for other indications, follow-up patients, and direct referral from the other hospital consultants. A hundred consecutive patients from each month over 2 years were evaluated to get a snapshot into the working of the breast clinic.

In our department, the symptomatic breast clinic functions as a 'one-stop-shop'. Most patients are given the diagnosis on the same day. All patients over 40 years get a mammogram as their first test, followed by a clinical examination or ultrasound if needed. Biopsies are performed on the same day.

The following variables were collected for the pandemic and pre-pandemic year for direct comparison:

- The total number of primary care referrals in a month

- The total number of patients seen in the 'one stop' clinics in a month

- The main presenting symptoms

- The median age of the patients

- Number of patients examined by their General Practitioners (GPs)

- The median waiting time to be seen by the specialist

- The total number of patients discharged from the 'one stop' clinics without a cancer diagnosis

- Number of patients discharged after imaging only

- Total number of biopsies

- Number of benign biopsies

- Number of cancers diagnosed

\section{Results}

A total of 516 patients were referred from primary care or GPs, and were seen in the Hospital 'one-stop Breast clinic' in April 2019. This number dropped to 330 patients during the peak of the pandemic in April 2020. Direct comparison to the mean data from the WCN showed the total mean monthly breast referrals for Wales dropped from 1717 in the pre-pandemic months to 810 in the pandemic month of April 2020. Hence, this reduction in primary referrals to the hospital during the pandemic's peak is in keeping with the approximately $47 \%$ reduction seen in Wales.

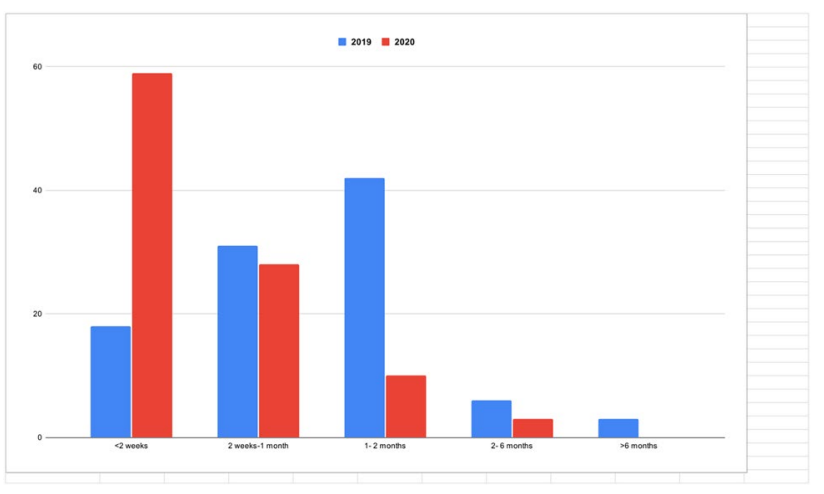

Fig. 1 Waiting time from GP to see a specialist over the 2 years

Figure 1 shows the trend of waiting times to see a specialist. In the pandemic month, most patients waited less than 2 weeks to see a specialist. This time compares to patients' median wait of 1-2 months before being seen by the specialist in 2019. Ninety percent of referrals from the GP were urgent suspected cancers or urgent referrals, and $10 \%$ were routine referrals. This trend of referrals did not change over two years. The number of patients examined by the GP dropped from 98 to $78 \%$ during the pandemic.

There was a reduction in patients over the age of 70 years during the pandemic year (3\%) compared to the pre-pandemic year $(19 \%)$ (Table 1). The number of patients under the age of 50 years was greater in the pandemic year.

During both years, there was no difference in the proportionate indication of referrals to the one-stop clinics [6]. However, over the 2 years, there was a slight difference. The majority (66\%) of referrals were due to breast lumps, followed by diffuse pain (13\%) in 2019. In 2020, the referral for breast lumps increased slightly (78\%); referrals for diffuse pain showed a reduction (3\%) (Table 2).

Table 3 gives a snapshot of the working of the breast clinic, with slight differences over the 2 years. The departmental policy is to undertake mammograms in all patients over 40 years of age before the clinician assessment. There were $7 \%$ and $12 \%$ of patients with no imaging in 2020 and 2019 , respectively. These patients were less than 40 years of age and were discharged based on clinical examination only.

Table 1 Age in years at presentation

\begin{tabular}{lll}
\hline Age (years) & $\begin{array}{l}\text { April } \\
2019 \\
(\%)\end{array}$ & $\begin{array}{l}\text { April } \\
2020 \\
(\%)\end{array}$ \\
\hline$<30$ & 16 & 18 \\
$\geq 30-39$ & 15 & 31 \\
$40-49$ & 24 & 33 \\
$50-69$ & 26 & 15 \\
$\geq 70$ & 19 & 3 \\
\hline
\end{tabular}


Table 2 Main presenting complaint at the GP/referral indication from the GP to the one-stop breast clinic

\begin{tabular}{lll}
\hline & Patients (\%) \\
\cline { 2 - 3 } $\begin{array}{lll}\text { Referral indication/ main presenting } \\
\text { complaint }\end{array}$ & $\mathbf{2 0 1 9}$ & $\mathbf{2 0 2 0}$ \\
\hline Breast lump & 66 & 78 \\
Axillary lump & 3 & 1 \\
Axillary tenderness & 0 & 1 \\
Nipple discharge & 2 & 5 \\
Nipple changes & 5 & 4 \\
Implant problem & 0 & 1 \\
Focal pain & 4 & 2 \\
Diffuse pain & 13 & 3 \\
Wound & 0 & 1 \\
Nodularity & 0 & 2 \\
Drawing sensation & 0 & 1 \\
Skin changes & 4 & 1 \\
Breast swelling & 1 & 0 \\
Supraclavicular lymph node & 1 & 0 \\
Heaviness & 1 & 0 \\
\hline
\end{tabular}

Approximately $75 \%$ of patients were discharged following a routine normal mammogram or ultrasound over the 2 years with no significant difference over the 2 years. The number of biopsies ranged from 10 (2019) to $18 \%$ (2020) over the 2 years.

Table 4 shows the diagnoses made following the biopsy. There was a 5\% and 7\% cancer diagnosis rate in 2020 and 2019 , respectively. In 2020, due to more younger patients presenting to the clinic, there were proportionately more fibroadenomas biopsies. The cancer diagnosis rate in symptomatic breast clinics is similar to historical data from Wales [7]. A review of data from WCN for the year 2019-2020 showed that out of all primary care referrals for breast cancer, $90 \%$ were reassured and discharged. Breast cancer data is in keeping with other cancers on the pathway for suspicion
Table 4 Diagnosis made following biopsy

\begin{tabular}{lll}
\hline Diagnoses based on biopsy results & 2019 & 2020 \\
\hline Normal & $\mathbf{0}$ & $\mathbf{2}$ \\
Fibroadenoma & $\mathbf{0}$ & $\mathbf{8}$ \\
Grade 1 invasive ductal carcinoma & $\mathbf{0}$ & $\mathbf{1}$ \\
Grade 2 invasive ductal carcinoma & $\mathbf{3}$ & $\mathbf{0}$ \\
Grade 3 invasive ductal carcinoma & $\mathbf{2}$ & $\mathbf{4}$ \\
Invasive lobular cancer & $\mathbf{1}$ & $\mathbf{0}$ \\
Grade 1 Invasive carcinoma- mucinous type & $\mathbf{1}$ & $\mathbf{0}$ \\
Cyst & $\mathbf{1}$ & $\mathbf{1}$ \\
Infection & $\mathbf{0}$ & $\mathbf{1}$ \\
Abscess & $\mathbf{0}$ & $\mathbf{1}$ \\
Scar tissue & $\mathbf{1}$ & $\mathbf{0}$ \\
Reactive changes & $\mathbf{1}$ & $\mathbf{0}$ \\
\hline
\end{tabular}

of cancer, where $88 \%$ are reassured and discharged, and $12 \%$ start treatment of cancer [5].

\section{Discussion}

This study highlights some interesting trends in the working of the one-stop breast clinics in the UK. One-stop breast clinics have been shown to reduce 'time- to- testing' and reduce patient anxiety, as patients receive 'on the day' diagnoses [8].

In this study, there was an overreliance on the urgent and 'urgent suspected cancer' risk category by GPs at $90 \%$, even though the cancer diagnosis rate remains at $5-7 \%$. This trend is not different to other cancers [9]. Moreover, many studies have shown that $70 \%$ of certain cancers were diagnosed in patients sent as routine referrals [10]. These trends underline the fact that differentiation of primary care referrals into risk categories is prone to errors. Multiple factors could be responsible for these trends, including vague symptoms, low sensitivity of clinical examination,
Table 3 Types of investigations at the one-stop breast clinic

\begin{tabular}{lll}
\hline Types of investigations at the clinic & Patients (\%) & $\mathbf{2 0 2 0}$ \\
\cline { 2 - 3 } & $\mathbf{2 0 1 9}$ & 7 \\
\hline Patients with no imaging & 12 & 34 \\
Patients undergoing US breast only & 26 & 6 \\
Patients undergoing mammograms only & 26 & 34 \\
Patients undergoing US and mammogram & 19 & 7 \\
Patients undergoing mammogram and US-guided core biopsy breast & 10 & 11 \\
Patients undergoing US-guided core biopsy only & 0 & 1 \\
Patients undergoing mammogram and US-guided biopsy axilla & 1 & 0 \\
Patients undergoing mammogram and US axilla & 3 & 0 \\
Patients undergoing US axilla only & 2 & 0 \\
Patients undergoing mammogram and punch biopsy & 1 & \\
\hline
\end{tabular}


fear of medicolegal issues leading to defensive medicine, high media sensitivity to missed diagnosis, and patients' requests for scans. The authors feel that it would be more accurate to see all patients in less than 2 weeks rather than differentiate referrals into risk categories. The current operational standard for NHS England is to assess 93\% of all its patients in the 2-week wait pathway [11].

The number of patients seen by the GP and the one-stop clinics dropped during the pandemic month. This reduction in numbers presenting to primary care was most likely due to the national message to stay at home, particularly for the elderly. Extrapolation from these numbers predicts that the future demand would shoot up due to pentup problems. The number of breast referrals were already $83-90 \%$ of the pre-pandemic levels in early 2021 [5]. Primary care referrals in Wales for all Cancers are $12 \%$ higher now than in 2019 [5].

There was a temporary reduction in the waiting time for patients to be seen in the symptomatic clinics during the pandemic to $<2$ weeks. This was most likely due to combination of factors including less number of primary referrals and staff re-organisation from screening to symptomatic services.

In this study, there was a reduction in the number of cancers diagnosed in the pandemic peak by $2 \%$. The reason for this is most likely due to less number of elderly patients presenting to the clinics and choosing to stay at home, following the national message to 'Stay at Home'. Our results are similar to a previous study [12], where the authors predicted 10 undiagnosed cancers in the community over the pandemic month accounting for between 69 and 100 additional new patient appointments per month. Crucially, the number of 'worried well' patients did not decrease during the pandemic as documented by the increase in the number of benign biopsies in this study.

The data from this study confirms increased reliance on imaging of some sort in the breast clinics, as $>90 \%$ of patients had imaging. Breast clinics use mammograms and ultrasound, which are cost-effective imaging modalities as they can be done quickly. In a separate report [13, 14], the authors have discussed ways to meet this demand for increased breast referrals by having 'straight to test' or 'imaging led' clinics running parallel to the traditional 'onestop clinics'. This arrangement would be particularly beneficial for most patients not requiring surgeons' input. They could be reassured and discharged without infringing on the surgeon's time. This way of working would require buy-in from both radiologists and surgeons but could be a more cost-effective use of precious radiologists and surgeon's time. This would lead to increased throughput of patients and introduce some flexibility within the working patterns [14, $15]$. More studies directly comparing the two ways of working would be beneficial, and could be easily implemented and incorporated in the recently promised post-COVID community Diagnostic Hubs.

There are certain limitations of this study. Small numbers and the retrospective nature of this analysis meant that statistical analysis could not be performed. However, this study only aimed to provide a snapshot of the functioning of the breast clinics to evaluate trends during the pandemic and predict future demand. There is also a potential of bias due to missing data and misclassification of symptoms due to subjectivity involved in the classification of symptoms. Authors hope this bias would be minimal as whenever possible the main presenting complaint was used for classification. Another limitation is that functioning of the breast clinic in a university hospital with particular staff members may not be applicable to other settings. However, the authors hope that the university setting involves varied staff members with varied experience and any discrepancy would iron out over the full month.

In conclusion, this study highlights that the referral patterns from primary care to specialists did not change during the pandemic, with most patients referred as 'urgent' or 'urgent suspected cancer'. The cancer diagnosis rate reduced from 7 to 5\% during the pandemic peak, but the number of 'worried well' patients did not reduce. The total number of referrals reduced, which is predictive of increased demand in the future. The authors have suggested ways to meet this demand.

Acknowledgements The authors would like to thank all the staff and patients of 'The Breast Centre', who helped to complete this study.

Author contribution Guarantor of integrity of the entire study - GJB, Study concept and design — GJB/ZS, Literature research — GJB/ZS, Clinical studies/data analysis - GJB/ZS, Statistical analysis - GJB/ ZS, Manuscript preparation — GJB/ZS, Manuscript editing — GJB/ZS

Data availability Yes.

\section{Declarations}

Conflict of interest The authors declare no competing interests.

\section{References}

1. World Health Organisation. Coronavirus - overview. http://www. who.int/health-topics/coronavirus . Accessed $1^{\text {st }}$ August 2021

2. World Health Organisation, Rolling updates on coronavirus (COVID-19). http://www.who.int/emergencies/diseases/novelcoronavirus-2019/events-as-they-happen. Accessed $1^{\text {st }}$ August 2021

3. Department of Health and Social Care. Stay at home.[Withdrawn] Coronavirus: stay at home, protect the NHS, save lives. http://www. gov.uk/government/publication/coronavirus-covid-19-informationleaflet/coronavirus-stay-at-home-protect-the-nhs-save-lives-webversion. Accessed $1^{\text {st }}$ August 2021 
4. Doughty J (2020) Statement from the Association of Breast Surgery, Statement. 2020. http://associationofbreastsurgery.org.uk/ for-members/covid-19-resources. Accessed $1^{\text {st }}$ August 2021

5. Observations of data used to measure the impact of COVID-19 on NHS Wales cancer services.

6. http://www.phwsharepoint.cymru.nhs.uk/whc/cancernetwork/ sitepages/delivery $\% 20$ Unit $\% 20$ scorecard.aspx. Accessed $1^{\text {st }}$ August 2021

7. National Institute of health and care excellence. Suspected cancer: recognition and referral. NICE Guideline NG12.London: NICE 2021. Accessed $1^{\text {st }}$ August 2021

8. Cochrane RA, Singhal H, Monypenny IJ, Webster DJ, Lyons K, Mansel RE (1997) Evaluation of general practitioner referrals to a specialist breast clinic according to the UK national guidelines. Eur J Surg Oncol 23(3):198-201. https://doi.org/10.1016/s07487983(97)92220-4 (PMID: 9236889)

9. Delaloge S, Bonastre J, Borget I, Garbay JR, Fontenay R, Boinon $\mathrm{D}$ et al (2016) The challenge of rapid diagnosis in oncology: diagnostic accuracy and cost analysis of a large-scale one-stop breast clinic. Eur J Cancer 66:131-7. https://doi.org/10.1016/j.ejca.2016. 06.021. PMID: 27569041
10. Robinson J, Hulbert A, Paul S, Howell T (2014) The two-week wait pathway: is it used appropriately? Eur Respiratory Journal 44(58):2739

11. Hamilton W, Coleman MG, Rubin G (2013) Colorectal cancer. BMJ 346:f3172

12. Department of Health. The likely impact of earlier diagnosis of cancer on costs and benefits to the NHS. London. 2011. Available at: https:// www.gov.uk/government/uploads/system/uploads/attachment_data/ file/213788/dh_123576.pdf. Accessed $1^{\text {st }}$ August 2021

13. Pearce BCS, Middleton H, Elakkad H, Schuster-Bruce A, Evans A, Clark SE (2021) The impact of COVID-19 in a breast service: patients attitudes towards accessing breast services during COVID19. https://doi.org/10.1308/rcsbull.2021.62

14. Bansal GJ (2019) Imaging led symptomatic breast clinics: a case for direct access to radiology. Br J Hosp Medicine (London) 80(7):377-79. https://doi.org/10.12968/hmed.2019.80.7.377

15. Bansal GJ, Chopra S (2021) Symptomatic breast services in the post-COVID era. Br J Hosp Med https://doi.org/10.12968/hmed. 2020.0679

Publisher's Note Springer Nature remains neutral with regard to jurisdictional claims in published maps and institutional affiliations. 